\section{Fusion Plasma as a Non-Equilibrium System}

\author{
Francesco Romanelli \\ Centro Ricerche Frascati, Italy
}

\author{
Carlos Hidalgo \\ Centro de Investigaciones Energéticas Medioambientales y Tecnológicas, Madrid, Spain
}

The heat and particle transport in fusion plasmas is generally due to turbulent processes associated with the presence of small scale instabilities (typical wavelengths of the order of the ion Larmor radius $\rho_{i}$ ) driven by the inhomogeneity of the density and temperature profiles in the direction normal to a magnetic surface. The magnitude of turbulent transport is probably the parameter affecting most the confinement properties and hence the economical performance of a fusion reactor. Thus, it is important to some extent to be able to control the turbulence level. This may occur in fusion plasmas, as well as many other complex systems, by selforganization phenomena which affect the turbulence level and the associated transport. During the last 15 years several plasma regimes caracterized by enhanced confinement have been experimentally reached, although only a few of them were obtained in steady state conditions. Thus, the achievement of these regimes in steady state conditions is an important line of research in controlled thermonuclear fusion.

The most important driving mechanisms for plasma turbulence were identified at the beginning of fusion research. Experimental investigations began systematically more than 20 years ago. As an example of the complexity of magnetizedplasma turbulence with respect to the conventional, neutral fluid turbulence, we consider the so called ion temperature gradient driven mode. The most important destabilization mechanism for such an instability is the so called 'negative compressibility', which is briefly described in the following. Let us consider a magnetic surface $S$, spanned by an equilibrium magnetic field $\mathbf{B}$, and equilibrium density and temperature spatial profiles $n_{\mathrm{o}}$ and $T_{\mathrm{o}}$ (for the sake of simplicity we will assume equal electron and ion temperatures) with gradients in the direction normal to $S$. Suppose that at time $t=0$ a perturbation $\phi$ in the electrostatic potential is produced. Due to their small mass the electrons will react immediately in order to shorten out the electric field $\mathrm{E}=-\nabla \phi$. By moving along the magnetic field lines they will produce an electron density perturbation $n_{e}=n_{\mathrm{o}}\left(e \phi / T_{\mathrm{o}}\right)$. Since this creates a local inbalance in the electric charge, the ion will also react in order to produce an ion density perturbation $n_{i}=n_{e}$, in such a way that local charge neutrality is restored. If we neglect the existence of a temperature gradient, the result of the original perturbation in the electric field will simply be the increase in the plasma pressure $\left(p=n_{e} T_{0}+n_{i} T_{\mathrm{o}}\right)$. Following such an increase, a restoring force associated with the plasma pressure will be produced and stable sound waves, propagating along the magnetic field, will be excited. The dynamics described up to this point is very similar to what happens in a neutral fluid. However, the situation changes dramatically in a magnetized plasma if a gradient in the ion temperature is considered. Due to the presence of the electric field the ions will move with a velocity $v_{i}$ such that the $\mathrm{E}+v_{i} \times \mathrm{B} \approx 0$, ie $v_{i} \approx \mathrm{E} \times \mathrm{B} / \mathrm{B}^{2}$.

The local value of the ion temperature will be perturbed by convection $\partial_{t} T_{i}=-v_{i} \nabla T_{0}$. Let us consider the situation at the time $t=\pi / 2 \omega$, with $\omega$ the frequency of the perturbation. Suppose that the phase velocity of the perturbation in the poloidal direction is such that the volume in which the original compression was produced coincides with the position of the local decrease of the ion temperature.
The global pressure change in that volume will be the sum of a positive contribution associated with the increase in the electron and ion density and a negative contribution associated with the decrease in ion temperature. If the net effect is a decrease of the local plasma pressure ( $\left.p=n_{e} T_{\mathrm{o}}+n_{i} T_{\mathrm{o}}+n_{\mathrm{o}} T_{i}<0\right)$, the compression will be amplified and an instability will be generated. Since the driving term is proportional to the ion temperature gradient, the instability takes the name of 'ion temperature gradient driven mode'.

A crude estimate of the turbulent thermal conductivity $\chi_{\text {turb }}$ (see Garbet, chapter 3.5) can be given on the basis of quasilinear theory as $\chi_{\text {turb }} \approx L_{r}{ }^{2} / \tau_{c}$ with $L_{r}$ being the radial correlation length and $\tau_{c}$ being the autocorrelation time of turbulence, of the order of the typical linear growth time of this class of instabilities $\tau_{c} \approx a / v_{t i}$, with $v_{t i}$ the ion thermal velocity and $a$ the minor radius. Thus, the reduction in $\chi_{\text {turb }}$ can be obtained either by an increase in $\tau_{c}$ or by a decrease in $L_{r}$. As an example of self organization mechanisms that lead to an increase in $\tau_{c}$ we mention the formation of large scale coherent structures, observed in several numerical simulations. These structures, which can be interpreted as solitary vortices, are coherent in the sense that their life time $\tau_{c}$ is much longer than the eddy turnover time. Thus, they behave as insulating structures and their occurrence is marked by a drop in the turbulent heat flux.

Direct experimental measurements and theoretical arguments show that plasma turbulence can manifest itself both by long and short radial correlation lengths. The turbulence radial correlation length $L_{r}$ can be determined by the competition between linear coupling and nonlinear decorrelation. The linear coupling acts in such a way as to produce radially elongated eddies. Indeed, the periodicity constraint in the poloidal and toroidal directions implies that a generic perturbation must have the form $\exp \{-i \omega t+i m \theta-i n \phi\}$ with $m$ and $n$ being integers. Such a perturbation is radially localized in a region of the order $\rho_{(, e, i)}$ around the magnetic surface where the pitch of the magnetic field lines is equal to $\mathrm{m} / \mathrm{n}$ (the so called rational surface). Since in an axisymmetric equilibrium the toroidal angle $\phi$ is an ignorable coordinate whereas the poloidal angle $\theta$ is not, a linear eigenmode is characterized by a single $n$ value and by the superposition of different poloidal harmonics with different $m$ values. The linear coupling between poloidal harmonics centred on neighbouring rational surfaces (ie the surfaces at which the 
pitch of the magnetic field lines is equal to $m / n$ and $(m \pm 1) / \mathrm{n})$ produces elongated eddies in the direction perpendicular to $S$. A rigorous analysis of the problem shows that the typical radial width of the eigenmode is of the order of $\left(a \rho_{i}\right)^{1 / 2}$. Thus, if the linear coupling dominates the radial correlation length can be estimated to be $L_{r} \approx$ $\left(a \rho_{i}\right)^{1 / 2}$. Nonlinear decorrelation mechanisms tend to break up this structure in such a way that each poloidal harmonic behaves independently from the others. If such a mechanism dominates we can estimate $L_{r} \approx \rho_{i}$. Therefore, the rough estimate of the local thermal conductivity given above can be reduced to two different limits: $\chi_{\text {turb }} \approx D_{\mathrm{B}}$ and $\chi_{\text {turb }} \approx\left(\rho_{t} / a\right) D_{\mathrm{B}}$, with $D_{\mathrm{B}}=c T / e \mathrm{~B}$ the Bohm coefficient. The two different limits are called Bohm and GyroBohm, respectively.

Historically, the first example of mechanism leading to the reduction of $L_{r}$, and therefore to a reduction of the turbulent flux, was proposed for the explanation of the transition to the High confinement mode (H-mode) which is obtained routinely in tokamaks with a magnetic separatrix at the plasma edge and corresponds to the formation of a local transport barrier. The improvement mechanism can be explained as the formation of a sheared $\mathbf{E} \times \mathbf{B}$ flow. If an electric field is produced in the direction perpendicular to the magnetic surface, the plasma starts to rotate with an $\mathbf{E} \times \mathbf{B}$ velocity which lies on the magnetic surface and is directed in the direction perpendicular to the equilibrium magnetic field. If the radial electric field is spatially nonhomogeneous, plasma volumes on neighbouring magnetic surfaces rotate with different velocities. Such a shear in the rotation velocity tears apart turbulent eddies elongated in the radial direction and reduces the transport level. Experimental support for this idea has been obtained in many magnetic confinement devices (ie tokamaks, stellarators and reversed field pinches) either by the analysis of spontaneous transitions or by using polarization probe techniques for the radial electric field generation. During the transition, the experimental measurements show the formation of an inhomogeneous radial electric field and the reduction of the turbulence level. But, how can such a field can be maintained? The radial electric field can be generated by the pressure gradient and poloidal and toroidal rotation. In the absence of substantial plasma rotation the radial electric field must be proportional to the local ion pressure gradient. Thus, a simple bifurcation paradigm can be constructed. A steepening of the pressure gradient produces a radial electric field which decreases the turbulence level and allows the maintaining of a steep pressure gradient. The closeness to turbulence thresholds, the type of instability driving the turbulence and the presence of sheared flows are considered critical elements in determining the transition between different transport regimes (ie Bohm versus Gyro-Bohm).

In the last five years a second mecha- nism for reducing the turbulent transport has been experimentally found in tokamaks which involves the production of nonmonotonic current density profiles (the current density is needed to produce the poloidal component of the equilibrium magnetic field). If such a condition is achieved, a transport barrier is formed with no turbulent transport. The understanding of this phenomenology is still in progress. A possible explanation might be that in these plasma regimes the magnetic configuration reduces the level of turbulence and/or the coupling between neighbouring poloidal harmonics. As a consequence, the power threshold for the transition to enhanced confinement regimes via sheared radial electric field effects is reduced. Thus, sheared radial electric fields could provide a universal mechanism to control plasma turbulence in fusion plasmas.

With an eye on the future, the understanding and development of methods for controlling plasma turbulence have opened a new path in plasma physics research and contributed to the design of a nuclear fusion plant.

\section{Further reading}

L.I. Rudakov, R.Z.Sagdeev Dokl. Akad. Nauk. SSSR 138 581 (1961)[ Sov. Phys. Dokl. 6415 (1961)]

M. Ottaviani, F. Romanelli, R. Benzi, M. Briscolini, P. Santangelo, S. Succi Phys. Fluids B2 671990

J.W. Connor, J.B. Taylor and H.R. Wilson Phys. Rev. Lett. 701903 (1993)

P.H. Diamond, T.S. Hahm Phys. Plasmas 23640 (1995)

\title{
The Understanding of Operational Limits
}

\author{
Laurent Villard \\ Ecole Polytechnique Fédérale de Lausanne, Switzerland
}

Given the high cost of experimental devices in magnetic fusion research it is important to have reliable and predictive tools for their operation. This, together with the complexity of the geometry, has prompted a major effort in the development of numerical techniques.

\section{From single particles...}

The particular magnetic geometry stems from the requirement that the particles should be confined by the magnetic field. The particle motion is a fast race along the field lines added to a slower drift across the magnetic surfaces. Therefore the topology is toroidal, with field lines helically twisting about the magnetic axis.

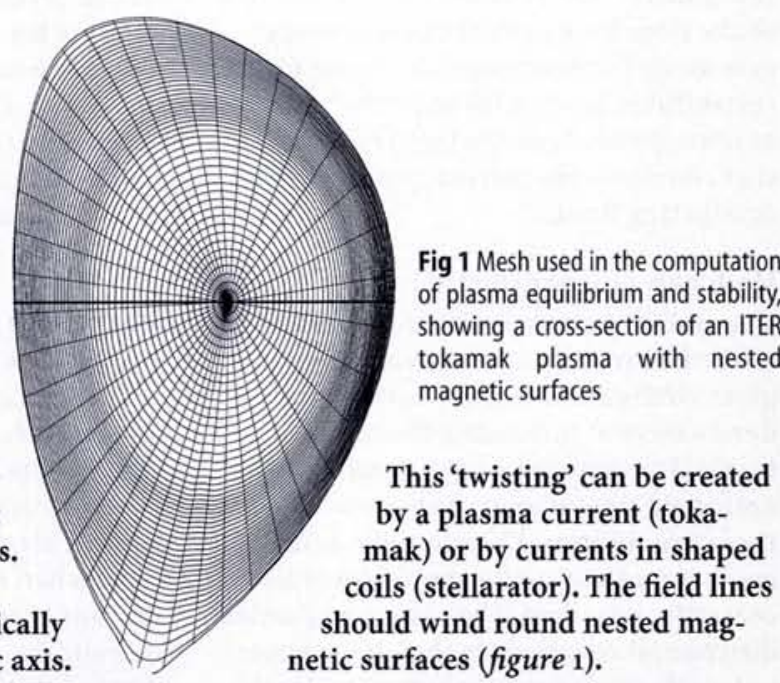

Fig 1 Mesh used in the computation of plasma equilibrium and stability, showing a cross-section of an ITER tokamak plasma with nested magnetic surfaces

This 'twisting' can be created by a plasma current (tokamak) or by currents in shaped (stellarator). The field lines netic surfaces (figure 1). 